\title{
Atividade inseticida da neolignana Burchelina sobre Oncopeltus fasciatus (Dallas) (Hemiptera: Lygaidae)
}

\section{Neolignan Burchellin insecticidal activity on Oncopeltus fasciatus (Dallas) (Hemiptera: Lygaidae)}

\author{
Denis Alves Monsores ${ }^{\dagger,}$, Thiago Dutra Dias ${ }^{\dagger}$, Igor Luiz Souza da Cruz ${ }^{\dagger, \bullet, \|, G a b r i e l a ~ A l v e s ~ M e l q u i ́ a d e s ~}$ \\ de Medeiros $^{\circ}$, José Maria Barbosa Filho ${ }^{\circ}$, Marise Maleck ${ }^{* \dagger, \S, \|}$
}

Como citar esse artigo. Monsores, D.A; Dias, T.D; Cruz, I.L.S; Medeiros, G.A.M; Filho, J.M.B; Maleck, M. Atividade inseticida da neolignana Burchelina sobre Oncopeltus fasciatus (Dallas) (Hemiptera: Lygaidae). Revista Teccen. 2021 Jan./Jun.; 14 (1): 02-06.

\section{Resumo}

Insetos praga são modelos de estudo na busca por métodos alternativos de controle que não agridam o homem e o meio ambiente, devido aos efeitos nocivos às plantações de grãos causando grandes impactos econômicos. $\mathrm{O}$ estudo buscou avaliar o potencial inseticida e ovicida da neolignana Burchelina sobre Oncopeltus fasciatus (Dallas) (Hemiptera: Lygaidae). Os bioensaios foram conduzidos no Laboratório de Insetos Vetores, da Universidade de Vassouras sobre ninfas de $5^{\circ}$ estádio, em triplicatas por grupo e com três repetições. Nas ninfas, a substância foi utilizada por tratamento tópico nas concentrações de 1, 10, 50, 100 e 200 $\mu \mathrm{g} / \mathrm{mL}$. Após o tratamento, as ninfas foram mantidas em temperatura ambiente, dieta habitual (água e semente de girassol) e observadas durante 20 dias. Os bioensaios sobre a massa de ovos foi realizada por aplicação tópica na concentração de $82 \mu \mathrm{g} /$ $\mathrm{mL}\left(\mathrm{DL}_{50}\right)$. Foram utilizados $20 \mathrm{mg}$ de ovos $/ 20 \mu \mathrm{L}$ da substância, em triplicata. O tratamento tópico com Burchelina resultou na mortalidade de ninfas de $5^{\circ}$ estádio em $69,2 \%(200 \mu \mathrm{g} / \mathrm{mL})$ e de $100 \%$ de toxicidade $(82 \mu \mathrm{g} / \mathrm{mL})$ sobre os ovos de $O$. fasciatus. Estes resultados demonstraram o potencial inseticida da Burchelina e sua importância no controle do Hemiptera Lygaidae.

Palavras-chave: Controle de vetores; Hemiptera; Ocotea cymbarum.

\begin{abstract}
Pest insects are study models in the search of alternative control methods that won't harm man or the environment, due to harmful effects caused to grain plantations resulting in great economic impacts. The study intended to evaluate the pesticide potential of neolignan Burchellin on the nymphs of Oncopeltus fasciatus (Dallas) (Hemiptera: Lygaidae). The experiment was conducted in the Vector Insects Laboratory of Vassouras University. The substance was used against $O$. fasciatus nymphs (5th instar) in triplicate per group with three repetitions, at $1-200 \mu \mathrm{g} / \mathrm{mL}$ final concentrations and applied by topic treatment $(\mu \mathrm{L} /$ nymph). After the treatment, the nymphs were kept in room temperature, normal diet (water and sunflower seed), and observed throughout 20 days. The bioassays on the egg $(20 \mathrm{mg} / 20 \mu \mathrm{L})$ were carried out by topical application at a concentration of 82 $\mu \mathrm{g} / \mathrm{mL}\left(\mathrm{LD}_{50}\right)$ in triplicate. Topical treatment with Burchellinresulted in the mortality of 5 th instar nymphs in $69,2 \%(200 \mu \mathrm{g} /$ $\mathrm{mL})$ and $100 \%$ toxicity $(82 \mu \mathrm{g} / \mathrm{mL})$ on $O$. fasciatus eggs. These results demonstrate the importance of studying neolignan in the control of Hemiptera Lygaidae.

Keywords: Vector control; Hemiptera; Ocotea cymbarum.
\end{abstract}

\section{Introdução}

O uso indiscriminado dos agrotóxicos no meio rural brasileiro tem sido a causa de efeitos nocivos para o meio ambiente e à saúde do trabalhador rural, que está constantemente em contato direto com essas substâncias (Almussa \& Schmidt, 2009). Diversos agravos à saúde são relacionados ao uso de agrotóxicos, como por exemplo, a ocorrência de anomalias congênitas, neoplasias, doenças mentais, infertilidade dentre outros, tornando-se um problema para a Saúde Pública (Cassal, Azevedo, Ferreira, Silva \& Simão, 2014). Para que uma substância química de efeito praguicida seja considerada ideal, deve ser efetiva em baixa concentração, apresentar ausência de toxicidade aos vegetais e demais mamíferos e ser biodegradável (Garcez, Garcez, Silva \& Sarmento, 2013).

As plantas possuem um sistema de defesa próprio que as protegem de outros vegetais, insetos fitófagos e herbívoros predadores. Estas defesas são substâncias químicas oriundas do metabolismo secundário da planta, as quais podem ser chamadas de fitoalexinas ou aleloquímicos (Pinto, Silva, Bolzani, Lopes \& Epifanio, 2002). Essas substâncias são farmacologicamente ativas, podendo apresentar atividade inseticida e servindo como uma alternativa de controle de pragas em culturas agrícolas ou casas e jardins, que têm implicações diretas na saúde única (Marangoni, Moura \& Garcia, 2012).

Afiliação dos autores:

${ }^{\dagger}$ Laboratório de Insetos Vetores, Universidade de Vassouras, Vassouras, RJ, Brasil.

* Pró-Reitoria de Ciências da Saúde, Universidade de Vassouras, Vassouras, RJ, Brasil.

- Doutorado Acadêmico em Biodiversidade e Saúde, Instituto Oswaldo Cruz, Fiocruz, Rio de Janeiro, RJ, Brasil.

§ Mestrado Profissional em Ciências Ambientais, Universidade de Vassouras, Vassouras, RJ, Brasil.

\| Laboratório de Entomologia Médica e Forense, Instituto Oswaldo Cruz, Fiocruz, Rio de Janeiro, RJ, Brasil.

${ }^{\circ}$ Instituto de Pesquisa em Fármacos e Medicamentos, Universidade Federal da Paraíba/UFPB, João Pessoa, PB, Brasil. 
A família Lauraceae compreende principalmente árvores e arbustos (Farago, Budel, Duarte \& Nakashima, 2005), composta por aproximadamente 50 gêneros e em torno de 2500 a 3000 espécies localizadas nas regiões tropicais e subtropicais do mundo (Van der Werff \& Richter, 1996). O gênero Ocotea possui cerca de 300 espécies (Farago et al., 2005), e tem sido bastante estudado devido a diversidade de compostos químicos bioativos, como por exemplo: alcaloides benzilisoquinolínicos e aporfínicos (Dias et al., 2003), lignanas (Morais et al., 1999) e neolignanas (Aiba, Braz Filho \& Gottlieb, 1973), monoterpenos (Diaz, Gottlieb \& Gottlieb, 1980), sesquiterpenos (Aiba et al., 1973) e os fenilpropanóides (Diaz et al., 1980).

Estudos demonstraram que a neolignana Burchelina isoladapela primeira vez dotronco de madeira de Aniba burchelli Kostern (Araujo Lima, Gottlieb \& Taveira Magalhães, 1972) apresentou efeitos nocivos contra diferentes insetos (Cabral, Garcia \& Kelecom, 1995; Nogueira, Mello, Kato \& Cabral, 2009; Narciso et al., 2014; Cabral, Alencar, Guimarães \& Kato, 2009) incluindo Oncopeltus fasciatus (Hemiptera: Lygaidae) (Cabral, Kelecom \& Garcia, 1999).

$\mathrm{O}$ inseto modelo de estudo Oncopeltus fasciatus Dallas, 1852 (Hemiptera: Lygaeidae) é encontrado nas regiões Centro-Oeste e Sul dos Estados Unidos (Feir, 1974). Este Hemiptera é utilizado como modelo experimental devido as suas características, morfologia, ciclo de vida curto e facilidade e manejo e criação (Andre, 1934). O. fasciatus se alimenta de Asclepias curassavica Linnaeus, planta conhecida popularmente como "oficial-de-sala", e é vetor de Phytomonas (Feir, 1974; Andre, 1934). As ninfas desse percevejo eclodem dos ovos e se desenvolvem através de 5 estádios de crescimento (ínstares), até alcançarem a fase adulta (Feir, 1974).

Este estudo buscou avaliar a atividade biológica da neolignana Burchelina, com relação ao ciclo do desenvolvimento e a ação inseticida, sobre Oncopeltus fasciatus.

\section{Material e Métodos}

\section{Isolamento de Burchelina}

A neolignana Burchelina (Figura 1) foi isolada de Ocotea cymbarum Kunth, popularmente conhecida como Louro-mamuri (Lima et al., 2011) ou Louro inamuí (Oliveira et al., 2008). Esta planta foi coletada nas proximidades de Belém, Pará, Brasil, e foi identificada pelo Prof. Klaus Kubiyzki, Universidade de Hamburgo, Hamburgo, Alemanha. A casca do caule seca e pulverizada $(253 \mathrm{~g})$ de O. cymbarum foi extraída primeiro com hexano e depois com diclorometano $\left(\mathrm{CH}_{2} \mathrm{Cl}_{2}\right)$ e finalmente etanol (EtOH). O solvente foi evaporado in vacum para produzir resíduos de hexano $(4,2 \mathrm{~g}), \mathrm{CH}_{2} \mathrm{Cl}_{2}(1,9 \mathrm{~g})$ e EtOH $(11,0 \mathrm{~g})$, respectivamente. $\mathrm{O}$ resíduo hexano foi cromatografado em uma coluna de sílica gel, e foi eluído com uma mistura de solventes: hexano contendo quantidades crescentes de $\mathrm{CHCl}_{3}, \mathrm{CHCl}_{3}$ com quantidades crescentes de $\mathrm{MeOH}$ e, finalmente, $\mathrm{MeOH}$ puro. As frações 3-5 que foram eluídas com hexano- $\mathrm{CHCl}$ 3 (1:1) produziram Burchelina $(411,9 \mathrm{mg}, 0,1628 \%)$ após purificação adicional por cromatografia em camada delgada preparativa. As estruturas foram identificadas usando análise espectroscópica e comparações com dados da literatura relacionados (Buechi \& Mak, 1977).

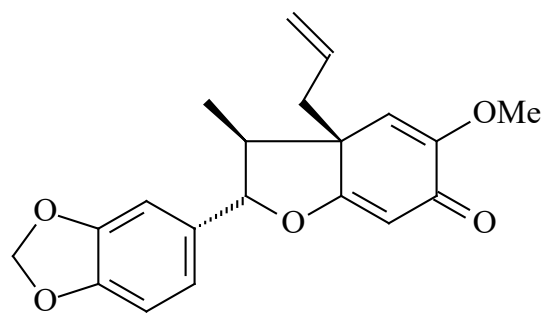

Figura 1. Burchelina, neolignana isolada de Ocotea cymbarum Kunth.

\section{Bioensaios}

Os bioensaios foram desenvolvidos no Laboratório de Insetos Vetores da Universidade de Vassouras, Rio de Janeiro, Brasil, em que foram utilizadas ninfas $(\mathrm{n}=10)$ de $5^{\circ}$ estádio de $O$. fasciatus por grupo teste, controle e controle testemunho, realizados em triplicata e três repetições. A colônia de insetos foi mantida em dieta habitual, de sementes de girassol triturada e água, e os insetos foram acondicionados em recipientes estéreis e a temperatura ambiente (Maleck, 2014). As ninfas utilizadas nos bioensaios, foram separadas da colônia com $24 \mathrm{~h}$ de antecedência para repouso e sem oferta de alimento.

A neolignana Burchelina foi dissolvida em dimetilsulfóxido(DMSO) nas concentrações de 1, 10, 50, 100 e $200 \mu \mathrm{g} / \mathrm{mL}$ e as soluções aplicadas por tratamento tópico na parte ventral do abdômen do inseto $(\mu \mathrm{L} /$ ninfa). O grupo controle testemunho recebeu tratamento tópico com $1 \mu \mathrm{L}$ de DMSO, e o grupo controle, recebeu tratamento tópico com $1 \mu \mathrm{L}$ de solução salina.

Após 1h da aplicação, os insetos foram colocados em um novo recipiente estéril, mantidos em dieta normal, de semente de girassol triturada e água, em temperatura ambiente. O desenvolvimento e a mortalidade dos insetos foram observados durante o período de 20 dias.

Para a avaliação da atividade ovicida, foram utilizados $20 \mathrm{mg}$ de ovos, em triplicata, no qual foi 
realizada a aplicação tópica de $20 \mu \mathrm{L}$ da solução, na concentração de $82 \mu \mathrm{g} / \mathrm{mL}\left(\mathrm{DL}_{50}\right)$, obtida através do tratamento tópico, à massa de ovos. Foram observados a taxa de ninfas que eclodiram e o número de insetos que atingiram a fase adulta.

Os dados foram analisados estatisticamente pela análise de variância (ANOVA) e teste de Tukey, através do software GraphPad InStat. No bioensaio com as ninfas e os adultos, a porcentagem de mortalidade foi calculada para cada tratamento e os valores corrigidos, utilizando a fórmula de Schneider-Orelli (Püntener, 1981).

\section{Resultados}

O tratamento tópico resultou na mortalidade de ninfas de $5^{\circ}$ instar em $46,1 \%(5,3 \pm 2,8)$ e $69,2 \%$ $(7,3 \pm 0,5)$ nas concentrações de $100 \mu \mathrm{g} / \mathrm{mL}$ e $200 \mu \mathrm{g} /$ $\mathrm{mL}$, respectivamente (Tabela 1). Quanto a mortalidade de adultos foi observada em 27,3\% (1,6 $\pm 1,1)$ na concentração de $100 \mu \mathrm{g} / \mathrm{mL}$ e $37,5 \%(1,0 \pm 0,0)$ na concentração de $200 \mu \mathrm{g} / \mathrm{mL}$ (Tabela 1).

$O$ percentual de ninfas que atingiram a metamorfose foi de $46,6 \%(4,6 \pm 2,8)$ na concentração de $100 \mu \mathrm{g} / \mathrm{mL}$ e apenas $26,6 \%(2,6 \pm 0,5)$ na concentração de $200 \mu \mathrm{g} / \mathrm{mL}$ (Tabela 1). O período de desenvolvimento das ninfas até a fase adulta foi de 3-8 dias, em $100 \mu \mathrm{g} /$ $\mathrm{mL}$, e 3-9 dias, em $200 \mu \mathrm{g} / \mathrm{mL}$ (Tabela 1).

Observou-se que algumas ninfas tratadas morreram durante a ecdise sem conseguir liberar a exúvia (mudas incompletas), resultando em 7\%, na concentração de $50 \mu \mathrm{g} / \mathrm{mL}$ e $3 \%$ nas concentrações de 10,100 e $200 \mu \mathrm{g} / \mathrm{mL}$ (Figura 2).

Tabela 1. Duração em dias do desenvolvimento, ecdises e mortalidade de Oncopeltus fasciatus tratados ( $5^{\circ}$ st) por uso tópico com Burchelina isolada de Ocotea cymbarum nas concentrações de 1, 10, 50, 100 e $200 \mu \mathrm{g} /$ $\mathrm{mL}$.

\begin{tabular}{cccccccccc}
\hline \multirow{2}{*}{ TRATAMENTO } & PERIODO (DIAS) & MUDA & \multicolumn{3}{c}{ MORTALIDADE } & \multicolumn{2}{c}{ MORTALIDADE } \\
& & & & & NINFAS & \multicolumn{2}{c}{ ADULTOS } \\
\hline \multirow{2}{*}{ Controle } & X DP & IV & X DP & $\%$ & X DP & $\%$ & X DP & $\%$ \\
\cline { 2 - 9 } DMSO & $7,5 \pm 2,0$ & $4-12$ & $9,6 \pm 0,5 \mathrm{a}$ & 96,6 & $0,3 \pm 0,5 \mathrm{a}$ & 3,3 & $1,0 \pm 1,0$ & 10,3 \\
1 & $7,5 \pm 1,9$ & $3-11$ & $8,6 \pm 0,5 \mathrm{ac}$ & 86,6 & $1,3 \pm 0,5 \mathrm{ac}$ & 13,3 & $1,0 \pm 1,0$ & 11,5 \\
10 & $7,3 \pm 0,7$ & $6-9$ & $6,0 \pm 1,0 \mathrm{ac}$ & 60 & $4,0 \pm 1,0 \mathrm{ac}$ & 30,8 & $0,6 \pm 1,1$ & 11,1 \\
50 & $6,9 \pm 1,4$ & $4-10$ & $7,0 \pm 1,7 \mathrm{ac}$ & 70 & $3,0 \pm 1,7 \mathrm{ac}$ & 19,2 & $2,0 \pm 2,6$ & 19,1 \\
100 & $7,0 \pm 1,6$ & $3-9$ & $6,0 \pm 2,0 \mathrm{ac}$ & 60 & $4,0 \pm 2,0 \mathrm{ac}$ & 30,8 & $3,0 \pm 2,0$ & 24,6 \\
200 & $6,0 \pm 1,6$ & $3-8$ & $4,6 \pm 2,8 \mathrm{bc}$ & 46,6 & $5,3 \pm 2,8 \mathrm{bc}$ & 46,1 & $1,6 \pm 1,1$ & 27,3 \\
& $7,3 \pm 1,9$ & $3-9$ & $2,6 \pm 0,5 \mathrm{bd} * *$ & 26,6 & $7,3 \pm 0,5 \mathrm{bd} *$ & 69,2 & $1,0 \pm 0,0$ & 29,3
\end{tabular}

Experimentos com 10 ninfas ( $5^{\circ}$ instar) de Oncopeltus fasciatus, para cada grupo teste e controle, em triplicatas e três repetições. Valores de média e desvio padrão $(\mathrm{X} \pm \mathrm{DP})$. Intervalo de Variação (IV). Valores seguidos da mesma letra não possuem diferenças significativas. Níveis de significância por Teste de Tukey estão representadas por $* * \mathrm{P}<0,01$ vs. controle testemunho de DMSO.

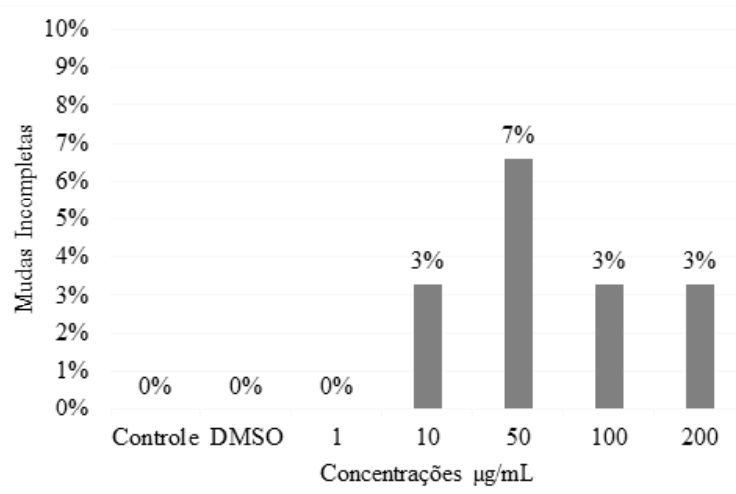

Figura 2. Percentual de mudas incompletas de $O$. fasciatus no tratamento tópico. 
A aplicação tópica da substância, sobre a massa de ovos de $O$. fasciatus, resultou em $2 \%$ de eclosão de ninfas, em comparação aos grupos controle (Tabela 2).

\section{Discussão}

A família Lauraceae émuito utilizada na medicina popular, e apresenta utilização variada, desempenhando diversas funções contra diferentes doenças, como antirreumática, depurativa, tônico, estomáquico, contra abcessos e, no uso externo sob feridas e úlceras. Algumas espécies de Lauraceae apresentam grande importância econômica, sendo utilizadas na culinária, fabricação de papel, construção civil, indústria farmacêutica e química (Betim, 2016).

O gênero Ocotea possui espécies de grande importância no setor de marcenaria, na construção civil, indústria química e farmacêutica, com utilização na medicina tradicional, apresentando ação antimicrobiana e antiparasitária (Betim, 2016; Araújo, 2012). Algumas espécies pertencentes ao gênero Ocotea apresentam atividade larvicida frente ao Aedes aegypti, destacando as espécies Ocotea pulchella (Araújo, 2012) e Ocotea cymbarum (Narciso, 2014).

$\mathrm{O}$ inseto modelo $O$. fasciatus apresentou sensibilidade à extratos hexânicos de Clusia fluminensis (Duprat et al., 2017). A substância isolada lanosterol mostrou resultados satisfatórios quanto a mortalidade de $O$. fasciatus, além disso, em relação ao desenvolvimento das ninfas em adultos, ocorreram atrasos significativos nos insetos tratados com este triterpeno (Duprat et al., 2017).

Neste estudo, os dados mostraram uma sensibilidade maior destes insetos na fase juvenil, que também foi observado em ninfas de $O$. fasciatus tratados topicamente com pinoresinol que resultou em $60 \%$ e $90 \%$ de mortalidade nas concentrações de 10 e $25 \mu \mathrm{g} /$ $\mathrm{mL}$, respectivamente (Cabral et al., 1999). A substância Burchelina demonstrou expressiva significância quanto à mortalidade de ninfas de $5^{\circ}$ estádio de $O$. fasciatus nas maiores concentrações, e atividade tóxica para os ovos de $O$. fasciatus. Os dados sugerem a contribuição da família laurácea no controle de insetos e redução de pragas.

\section{Considerações Finais}

A neolignana Burchelina mostrou-se eficaz quanto a mortalidade de ninfas e efeito ovicida contra $O$. fasciatus, atuando diretamente no seu ciclo de vida, o que sugere importante fonte de estudo no controle de insetos fitófagos.

\section{Agradecimentos}

O presente estudo foi realizado com apoio da Fundação Educacional Severino Sombra (FUSVE); o apoio da Fundação de Amparo à Pesquisa do Estado do Rio de Janeiro - FAPERJ, pela bolsa PIBIC/FAPERJ; e com apoio da Coordenação de Aperfeiçoamento de Pessoal de Nível Superior - Brasil (CAPES) - Código de Financiamento 001.

\section{Referências}

Aiba, C. J., Braz Filho, R., Gottlieb, O. R. (1973). Porosin: a neolignan from Ocotea porosa. Phytochemistry, 12(2), 413-416.

Almussa, A., Schmidt, M. L. G. (2009). O contato com agrotóxicos e os possíveis agravos à saúde de trabalhadores rurais. Revista de Psicologia da

Tabela 2. Tratamento tópico com Burchelina isolada de Ocotea cymbarum na concentração de $82 \mu \mathrm{g} / \mathrm{mL}$, em massa de ovos de Oncopeltus fasciatus.

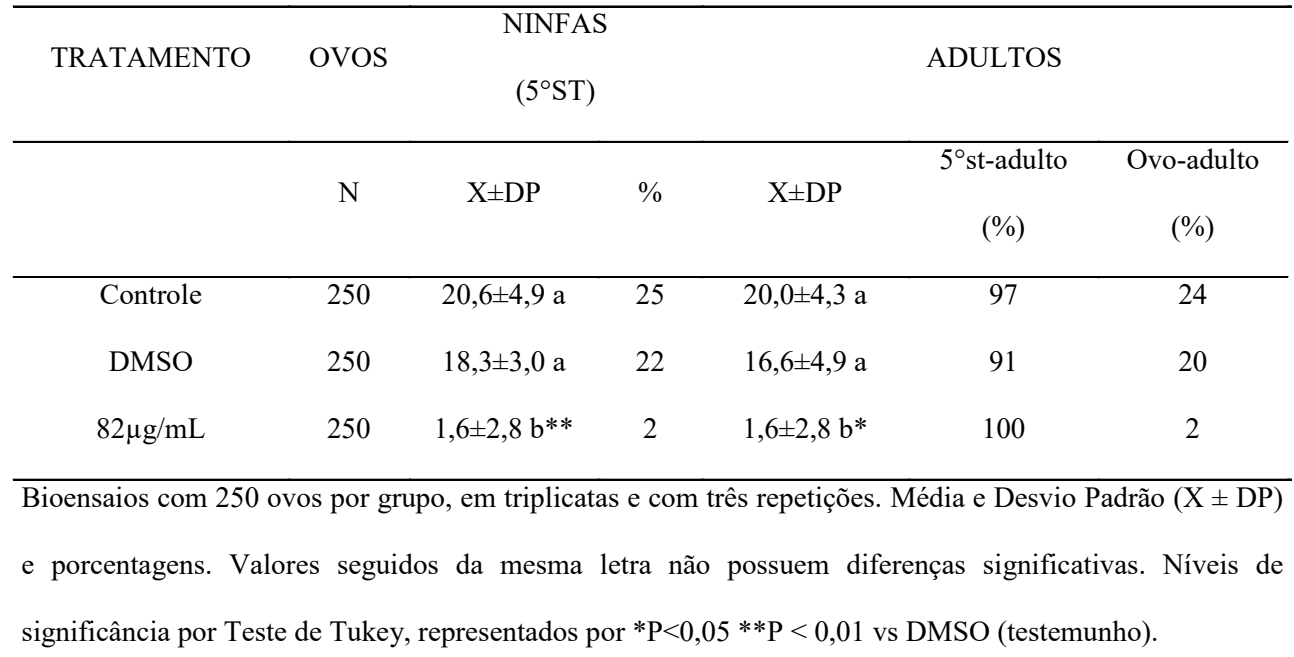




\section{UNESP, 8(2), 184-188.}

Andre, F. (1934). Notes on the biology of Oncopeltus fasciatus (Dallas). Iowa State Coll J Sci,9(1), 73-87.

Araújo, A. C. R. (2012). Estudo fitoquímico e avaliação das atividades alelopática, antioxidantes, antilarvicidas e citotoxicidade das folhas de Ocotea pulchella Mart. (Lauraceae). Dissertação de Mestrado, Universidade Federal do Paraná.

Araújo-Lima, O., Gottlieb, O. R., Magalhães, M. T. (1972). Burchellin, a neolignan from Aniba burchelli. Phytochemistry, 11, 2031.

Betim, F. C. M. (2016). Estudo fitoquímico e biológico da espécie Ocotea nutans (Nees) Mez, Lauraceae. Dissertação de Mestrado, Universidade Federal do Paraná.

Buechi, G., Mak, C. P. (1977). Biomimetic syntheses of the neolignans guaianine, burchellin, 2-epi, 3a-epiburchellin and futoenone. Journal of America Chemical Society, 99, 8073-8075.

Cabral, M. M. O., Alencar, J. A., Guimarães, A. E., Kato, M. J. (2009). Larvicidal activity of grandisin against Aedes aegypti. Journal of the American Mosquito Control Association, 25(1), 103-105.

Cabral, M. M. O., Azambuja, P., Gottlieb, O., Kleffmann, T., Garcia, E., Schaub, G. (2001). Burchellin: effects on Triatoma infestans and on Trypanosoma cruzi within this vector. Parasitology Research, 87(9), 730735 .

Cabral, M. M. O., Garcia, E. S., Kelecom, A. (1995). Lignanes from the Brazilian Melia azedarach, and their activity in Rhodnius prolixus (Hemiptera, Reduviidae). Memórias do Instituto Oswaldo Cruz, 90(6), 759763.

Cabral, M. M. O., Kelecom, A., Garcia, E. S. (1999). Effects of the lignan, pinoresinol on the moulting cycle of the bloodsucking bug Rhodnius prolixus and of the milkweed bug Oncopeltus fasciatus. Fitoterapia, 70(6), 561-567.

Cabral, M. M. O., Mendonça, P. M., Gomes, C. M. S., Barbosa-Filho, J. M., Dias. C. S., Soares, M. J., Queiroz, M. M. C. (2007). Biological activity of yangambin on the postembryonic development of Chrysomya megacephala (Diptera: Calliphoridae). Journal of Medical Entomology, 44(2), 249-255.

Cassal, V. B., Azevedo, L. F., Ferreira, R. P., Silva, D. G., Simão, R. S. (2014). Agrotóxicos: uma revisão de suas consequências para a saúde pública. Revista Eletrônica em Gestão, Educação e Tecnologia Ambiental, $18(1), 437-445$.

Diaz, A. M. P., Gottlieb, H. E., Gottlieb, O. R. (1980). Dehydrodieugenols from Ocotea cymbarum. Phytochemistry, 19(4), 681-682.

Dias, C. S., Silva, I. G., Cunha, E. V. L., Silva, M. S., Braz-Filho, R., Barbosa-Filho, J. M. (2003). Isolamento e identificação de novos alcalóides de Ocotea duckei Vattimo (Lauraceae). Revista Brasileira de Farmacognosia, $13,62-63$.

Duprat, R. C., Anholeti, M. C., Sousa, B. P. D., Pacheco, J. P. F., Figueiredo, M.R., Kaplan, M.A., Santos, M.G., Gonzalez, M. S., Ratcliffe, N. A., Mello, C. B., Paiva, S. R., Feder, D. (2017). Laboratory evaluation of Clusia fluminensis extracts and their isolated compounds against Dysdercus peruvianus and Oncopeltus fasciatus. Revista Brasileira de Farmacognosia, 27(1), 59-66.

Farago, P. V., Budel, J. M., Duarte, M. R., Nakashima, T. (2005). Análise morfoanatômica de folhas de Ocotea puberula (Rich.) Nees, Lauraceae. Revista Brasileira de Farmacognosia, 15, 250-255.

Feir, D. (1974). Oncopeltus fasciatus: a research animal. Annual Review of Entomology, 19(1), 81-96.

Garcez, W. S., Garcez, F. R., da Silva, L. M., Sarmento, U. C. (2013). Substâncias de origem vegetal com atividade larvicida contra Aedes aegypti. Revista Virtual de Química, 5(3), 363-393.

Lima, R. B. D. A., Silva, J. A. A. D., Marangon, L. C., Ferreira, R. L. C., Silva, R. K. S. (2011). Sucessão ecológica de um trecho de Floresta Ombrófila Densa de Terras Baixas, Carauari, Amazonas. Pesquisa Florestal Brasileira, 31(67), 161-172.

Marangoni, C., Moura, N. F., Garcia, F. R. M. (2012). Utilização de óleos essenciais e extratos de plantas no controle de insetos. Revista de Ciências
Ambientais, 6(2), 95-112.

Maleck, M., Serdeiro, M. T., Santos, F., Braun, A., Chaves, D., Faria, A., et al. (2014). Bioactivity of Brazilian plant extracts on Oncopeltus fasciatus. International Journal of Fauna and Biological Studies, 1(6), 114-120.

Morais, L. C. S. L., Almeida, R. N., Cunha, E. V. L., Silva, M. S., BarbosaFilho, J. M., Gray, A. I. (1999). Further lignans from Ocotea duckei. Pharmaceutical Biology, 37(2), 144-147.

Narciso, J. O. A., Soares, R. O. A., Mallet, J. R. S., Guimarães, A. É., Chaves, M. C. O., Barbosa-Filho, J. M., Maleck, M. (2014). Burchellin: study of bioactivity against Aedes aegypti. Parasites \&Vectors, 7(1), 172.

Nogueira, C. D. R., Mello, R. P., Kato, M. J., Cabral, M. M. O. (2009). Disruption of Chrysomya megacephala growth caused by lignan grandisin. Journal of Medical Entomology, 46(2), 281-283.

Oliveira, A. C. M., Nepstad, D. C., McGrath, D. G., Silva, A. F. (2009). Impactos ecológicos do manejo comunitário de recursos naturais em comunidades ribeirinhas de várzea e terra firme na região de Tefé, AM. Novos cadernos NAEA, 11(2), 57-84.

Pinto, A. C., Silva, D. H. S., Bolzani, V. S., Lopes, N. P., Epifanio, R. A. (2002). Produtos naturais: atualidade, desafios e perspectivas. Química Nova, 45-61.

Püntener, W. (1981) Manual for field trials in plant protection second edition. Agricultural Division, Ciba-Geigy Limited.

Van der Werff, H., Richter, H. G.(1996). Toward an improved classification of Lauraceae. Annals of the Missouri Botanical Garden, 83(3), 409-418 